\title{
Flexoelectricity and competition of time scales in electroconvection
}

\author{
Tibor Tóth-Katona, Nándor Éber, Ágnes Buka \\ Research Institute for Solid State Physics and Optics, \\ Hungarian Academy of Sciences, H-1525 Budapest, P.O.B. 49, Hungary \\ Alexei Krekhov \\ Physikalisches Institut, Universität Bayreuth, D-95440 Bayreuth, Germany
}

(Dated: October 29, 2018)

\begin{abstract}
Novel behavior in electroconvection (EC) has been detected in nematic liquid crystals (NLCs) under the condition of comparable timescales of the director relaxation and the period of the driving ac voltage. The studied NLCs exhibit standard EC (s-EC) at the onset of the instability, except one compound in which nonstandard EC (ns-EC) has been detected. In the relevant frequency region, the threshold voltage for conductive s-EC bends down considerably, while for dielectric s-EC it bends up strongly with the decrease of the driving frequency. We show that inclusion of the flexoelectric effect into the theoretical description of conductive s-EC leads to quantitative agreement, while for dielectric s-EC a qualitative agreement is achieved. The frequency dependence of the threshold voltage for ns-EC strongly resembles that of the dielectric s-EC.

PACS numbers: 47.54.-r, 61.30.Gd, 47.20.Lz
\end{abstract}

\section{INTRODUCTION}

Electroconvection (EC) in nematic liquid crystals (NLCs) is an extensively studied example of electric field induced instabilities [1, 2, 3]. It also serves as a convenient model system for investigating pattern forming phenomena in complex, anisotropic fluids driven out of equilibrium.

NLCs have a uniaxial orientational order which is represented by the unit vector $\mathbf{n}$, the director. Consequently, NLCs possess direction dependent physical properties. The dielectric anisotropy $\varepsilon_{a}=\varepsilon_{\|}-\varepsilon_{\perp}$ and the conductivity anisotropy $\sigma_{a}=\sigma_{\|}-\sigma_{\perp}$ are two key parameters in EC ( $\|$ denotes the value along, and $\perp$ perpendicular to the director). While $\varepsilon_{a}$ governs the electric torque acting on the director in the presence of electric fields, the director distortions lead to an elastic restoring torque exerted on $\mathbf{n}$. Furthermore, the uniaxial symmetry of NLCs allows for a coupling between director orientation and flow, characterized by the 5 independent viscosity coefficients, resulting in a viscous torque if velocity gradients and/or temporal variation of $\mathbf{n}$ are present.

Electroconvection has most commonly been observed in planarly oriented ( $\mathbf{n}$ parallel to the substrates) layers of nematics with $\varepsilon_{a}<0$ and $\sigma_{a}>0$, though it shows up at some other combinations of $\varepsilon_{a}, \sigma_{a}$ and initial director orientation too [3]. In experiments usually samples of $10 \mu \mathrm{m} \lesssim d \lesssim 100 \mu \mathrm{m}$ thickness have been used. EC appears at a threshold rms value $U_{\mathrm{c}}$ of the applied ac voltage $U$ of frequency $f$ as a pattern consisting of dark and bright stripes with wavevector $\mathbf{q}$ when viewed in a microscope. EC patterns have great morphological richness at the onset: normal rolls (q parallel with the initial $\mathbf{n}$ ) and oblique rolls (q encloses a finite angle with $\mathbf{n}$ ), stationary as well as traveling patterns have been detected depending on material parameters and on the driving frequency.
Detailed theoretical studies in the past decades have established a firm understanding of this pattern forming phenomenon, combining the equations of nematohydrodynamics (anisotropic Navier-Stokes equation for the flow plus the balance of torques acting on the director) with those of electrodynamics assuming that the NLC has an ohmic electrical conductivity. First it has been shown using a simple one-dimensional approximation that the EC pattern is excited by the Carr-Helfrich feedback mechanism [4, 5]: spatial director modulations induce a separation of space charges due to the anisotropic conductivity; electrostatic forces induce a flow in form of vortices; flow exerts a destabilizing viscous torque on the director against restoring elastic and electric torques. When this feedback becomes positive (at $U \geq U_{\mathrm{c}}$ ) a specific director fluctuation grows up; i.e., a periodic director modulation, characterized by a finite tilt angle with respect to the initial alignment, develops. As the director is the optical axis of the NLC, the tilt leads to a modulation of the refractive indices. The pattern becomes optically detectable either with or without a polarizer due to light focusing/defocusing effects (shadowgraph image [6]) as well as via birefringence if crossed polarizers are used. The complex physical phenomena involved in the mechanism occur on three different time scales, characterized by the director relaxation time $\tau_{\mathrm{d}}=\frac{\gamma_{1} d^{2}}{K_{11} \pi^{2}}$, the charge relaxation time $\tau_{\mathrm{q}}=\frac{\epsilon_{0} \epsilon_{\perp}}{\sigma_{\perp}}$ and the viscous relaxation time $\tau_{\mathrm{v}}=\frac{\rho d^{2}}{\alpha_{4} / 2}$. Here $K_{11}$ is the splay elastic modulus, $\rho$ is the density, $\gamma_{1}$ is the rotational, $\alpha_{4} / 2$ the isotropic viscosity. Typically $\tau_{\mathrm{d}} \gg \tau_{\mathrm{q}} \gg \tau_{\mathrm{v}}$ [1]. For example, in a $d=20 \mu \mathrm{m}$ sample of nematic Phase 5 (Merck \& Co., Inc.) with a typical conductivity of $\sigma_{\perp}=8.2 \times 10^{-8}(\Omega \mathrm{m})^{-1}$ at $T=30^{\circ} \mathrm{C}$, one has $\tau_{\mathrm{d}}=0.46 \mathrm{~s}, \tau_{\mathrm{q}}=5.6 \times 10^{-4} \mathrm{~s}$ and $\tau_{\mathrm{v}}=1.4 \times 10^{-5} \mathrm{~s}$.

Though the 1-d theoretical description managed to capture the essence of the instability mechanism, it rep- 
resents a substantial simplification of the problem, therefore the applicability of the resulting (approximate) analytical formulas are limited. This has necessitated later the development of a comprehensive 3-d theory, composed of six coupled partial differential equations (PDEs), known today as the standard model (SM) of EC [7]. The equations can be conveniently made nondimensional by measuring lengths in units of $d / \pi$ and time in units of $\tau_{\mathrm{d}}$. Then via a linear stability analysis the $\mathrm{SM}$ can provide the frequency dependence of the threshold voltage $U_{\mathrm{c}}(f)$, that of the critical wave vector, as well as the spatiotemporal dependence of the director field, the velocity field and the charge distribution at onset. According to the model, a finite threshold of the primary instability in planar geometry requires a positive $\sigma_{a}$ and a negative (or slightly positive) $\varepsilon_{a}$. Under these conditions, depending on the driving frequency $f$, two different solution types exist. Below the crossover frequency $f_{\mathrm{c}}$, in the conductive regime, the charge distribution oscillates with $f$, while the director field is stationary in leading order. In this regime, SM gives two solutions with different spatiotemporal symmetry (mode I and mode II), however, for the threshold behavior only the solution with the lower $U_{\mathrm{c}}$ (mode I) is relevant [8, 9]. Above $f_{\mathrm{c}}$, in the dielectric regime, the situation is reversed: the charge distribution is stationary in leading order, while the director oscillates with $f$. Here, the model also yields two solutions (mode III and mode IV), but the threshold behavior is described by mode III which has lower $U_{\mathrm{c}}$ compared to mode IV. These electroconvection scenarios, that are captured by the SM, will be referred to as standard electroconvection (s-EC). Various predictions of the SM have been compared with experimental results obtained on a number of NLCs and usually an excellent agreement has been found - see, e.g., [2, 10, 11].

In s-EC, the conductive and the dielectric regimes correspond to two competing pattern forming modes which have different $U_{\mathrm{c}}(f)$ and $U_{\mathrm{c}}(d)$ characteristics. The frequency dependence of $U_{\mathrm{c}}$ and $q_{\mathrm{c}}$ has a concave (diverging) shape in the conductive regime whereas they have a square root-like behavior in the dielectric one. As far as the thickness dependence is concerned, an inspection of the non-dimensional equations of the SM shows that by scaling only 5 of the 6 PDEs became invariant with respect to $d$; the equation for the potential still has an explicit $d$ dependence via a factor $\tau_{\mathrm{d}} / \tau_{\mathrm{q}} \propto \sigma_{\perp} d^{2}-$ see e.g., equations (A6)-(A11) in [12]. Nevertheless, in the conductive regime, $U_{\mathrm{c}}$ is thickness independent in the lowest order time Fourier approximation for the experimentally most relevant $(10-100 \mu \mathrm{m})$ thickness range, where $\tau_{\mathrm{d}} \gg \tau_{\mathrm{q}}$ [7]. In contrast, in the dielectric regime an analogous approximation gives $U_{\mathrm{c}} \propto d$.

At typical $(d \geq 10 \mu \mathrm{m})$ cell thicknesses the conductive regime appears at low frequencies and the dielectric rolls are observable at higher $f$. The change of pattern type occurs at $f_{\mathrm{c}}$ which depends significantly on $\sigma_{\perp}$ as well as on $d$. Therefore, in order to extend the frequency range of the dielectric regime, thus to shift $f_{\mathrm{c}}$ to lower values, one has to reduce either the cell thickness or the electric conductivity or both. When both $\sigma_{\perp}$ and $d$ are small enough, the conductive regime does not occur at all.

The SM regards NLCs as regular dielectrics, where the source of the electric polarization is the electric field. In NLCs, however, flexoelectric polarization [13, 14, 15] may appear even in the absence of the electric field, if the director field is distorted. Flexoelectricity produces an additional contribution to the charge distribution and to the electric torque acting on the director. It is also known for a long time that under large enough dc voltage, flexoelectricity may distort the director field in the planar geometry resulting in a non-convective striped pattern oriented parallel with the initial $\mathbf{n}$ [16]. The threshold voltage and the wavenumber of this non-convective pattern, as well as the frequency dependence of the threshold characteristics have been calculated [17, 18].

The effect of flexoelectricity on EC has also been studied and it has been shown that for the commonly used nematic material parameters, and for a typical sample thickness of $d \gtrsim 10 \mu \mathrm{m}$, in the usually studied (not too low) frequency range of conductive s-EC, flexoelectricity has no significant influence on the characteristics at the onset of instability [19, 20, 21]. That gives the reason why the contribution of the flexoelectric polarization has usually been neglected in the SM. On the other hand, it has also been shown that when applying a dc voltage, the EC threshold becomes independent of $d$ and $\sigma_{\perp}$ [19]. Moreover, flexoelectricity leads to an appreciable reduction of the dc EC threshold and it also influences the direction of the rolls [20]. Obviously, there must be an ac frequency range in which the effect of flexoelectricity diminishes with the increase of the frequency. This frequency range, however, has not yet been studied in details.

In the extended SM (i.e., with the inclusion of the flexoelectric polarization) two additional parameters, the flexoelectric coefficients $e_{1}$ and $e_{3}$ [8, 13] come into play. An analysis of the nematohydrodynamic equations linearized around the basic state has proven that flexoelectricity establishes a coupling between the conductive and dielectric modes introduced above [8, 12] resulting in a complex time dependence. The solutions still can be classified according to their spatiotemporal parity [12]. The even parity solution has mode I coupled to mode IV (mode I+IV), and corresponds to the "conductive regime", while in the odd parity "dielectric" solution mode III couples to mode II (mode II+III) [22].

What has been said above, applies to substances with material parameters satisfying $\varepsilon_{a}<0, \sigma_{a}>0$ or $\varepsilon_{a}>0$, $\sigma_{a}<0$ with the appropriate boundary conditions [3]. In contrary, in case of nematics with $\varepsilon_{a}<0$ and $\sigma_{a}<0$ [3, 14], where the feedback loop remains negative for all voltages, the Carr-Helfrich mechanism excludes the existence of EC patterns. Nonetheless, a convection roll pattern has long ago been observed in such compounds in ac electric field [23, 24] and has been reconsidered systematically recently in a few nematics [25, 26, 27]. Some basic 
characteristics of these patterns, like the orientation of the rolls (nearly parallel with the initial $\mathbf{n}$ ), the contrast, the frequency dependence of $U_{\mathrm{c}}$ and that of the critical wave number have been found considerably different from those of the s-EC. Since this kind of pattern formation is not predicted by the classical SM (without the incorporation of the flexoelectricity), it has been called nonstandard electroconvection (ns-EC) 25]. Recently, convection patterns with similar properties have been detected in nematics built up from bent-core molecules [28, 29, 30] that may exhibit giant flexoelectricity [31].

These experiments have triggered a recent reconsideration of the role of flexoelectricity within the extended $\mathrm{SM}$ for a wider range of material parameters. It has been shown that in some cases flexoelectricity cannot be disregarded. At high frequencies, in the dielectric regime a nonzero $e_{1}$ and $e_{3}$ leads to a considerable decrease of $U_{\mathrm{c}}$ [32]. What is even more important, the flexoelectric contribution to the charge separation yields finite instability threshold for planar nematics with $\sigma_{a}<0$ and $\varepsilon_{a}<0$, thus giving an explanation for ns-EC [12].

Special behavior can be expected (some might go beyond the applicability limit of the SM) in the parameter ranges where the period of the driving frequency $1 / f$ becomes comparable with one of the characteristic times of the system $\tau_{\mathrm{d}}, \tau_{\mathrm{q}}$ or $\tau_{\mathrm{v}}$. The majority of the experimental and theoretical studies of EC so far apply to relatively thick cells of medium conductivity, thus they do not extend to this range. We aim to fill this gap and we also show that flexoelectricity plays a crucial role in those ranges.

In the present paper we report on experimental studies and numerical simulations of EC aiming to approach the characteristic times of the system by $1 / f$, especially concentrating on $\tau_{\mathrm{d}}$ and in some special cases on $\tau_{\mathrm{q}}\left(\tau_{\mathrm{v}}\right.$ is usually very short, to approach it would require very thick cells and high driving frequencies, which would result in high threshold voltages not accessible experimentally). We focus on the frequency dependence of the threshold voltage $U_{\mathrm{c}}(f)$. The measurements have been carried out on five different nematic compounds: four of them exhibit s-EC, while in the fifth one, both s-EC and ns-EC patterns could be observed. The numerical analysis has been based on the extended standard model of EC which includes the flexoelectric effects [8, 12].

The structure of the paper is organized according to the pattern type in the low frequency range. After introducing the substances, the setup, and some details of the numerical analysis in Section II Section III is devoted to results on compounds in which conductive s-EC has been detected at low frequencies. In Section IV we discuss samples which exhibit dielectric s-EC at low $f$, while Section $\mathrm{V}$ describes the ns-EC instability. Finally, we conclude the paper with the discussion in Section VI.

\section{SUBSTANCES, EXPERIMENTAL SET-UP, AND DETAILS OF CALCULATIONS}

Five different NLCs have been used in the measurements. The three commercial nematic mixtures, Phase 5, Phase 5A and Phase 4 (from Merck \& Co., Inc.) as well as the 4-methoxy-benzylidene-4'-n-butyl-aniline (MBBA) have $\sigma_{a}>0$ and $\varepsilon_{a}<0$; so they exhibit s-EC upon application of an electric voltage. All measurements presented here on these compounds have been performed at $T=30^{\circ} \mathrm{C}$.

The fifth studied compound was the 4-n-octyloxyphenyl-4-n'-heptyloxy-benzoate (8/7) [33] which has the phase sequence: isotropic $-92{ }^{\circ} \mathrm{C}-$ nematic $-72.5{ }^{\circ} \mathrm{C}$ - smectic $\mathrm{C}-62^{\circ} \mathrm{C}$ - crystalline. It has $\varepsilon_{a}<0$ over the whole nematic temperature range, but shows a sign inversion from $\sigma_{a}<0$ to $\sigma_{a}>0$ as the temperature is increased toward the clearing point. Consequently, in this particular compound both ns-EC and s-EC could be investigated (although at different temperatures).

Our aim is to study the EC onset behavior in the vicinity of the characteristic relaxation times of the system; in order to approach that we have varied the cell thickness and the conductivity to tune $\tau_{\mathrm{d}}$ and $\tau_{\mathrm{q}}$.

The nematic liquid crystals have been enclosed between two parallel glass plates coated with etched transparent indium tin oxide (ITO) electrodes. Rubbed polyimide has been used to obtain a planar alignment. Cells in the thickness range from $d \approx 3 \mu \mathrm{m}$ to $d=40 \mu \mathrm{m}$ have been prepared. A spectrophotometer has been used for measuring $d$. It also enabled a systematic mapping of the thickness of the empty cell, thus determining its total variation throughout the whole active area (region between electrodes) of the sample. Typically, we have found a variation of $\pm 0.5 \mu \mathrm{m}$. The conductivity range extended from $10^{-9}(\Omega \mathrm{m})^{-1}$ to $10^{-7}(\Omega \mathrm{m})^{-1}$. The direction of the director at the surfaces is chosen as the $x$-axis. An electric field across the sample (along the $z$-axis) has been generated by applying a sinusoidal ac electric voltage of frequency $f$ and amplitude $\sqrt{2} U$ to the electrodes. The cells have been placed into an oven (an Instec hotstage) thermostatted within $\pm 0.05^{\circ} \mathrm{C}$.

EC patterns have been studied with polarizing microscopy using either the shadowgraph (single polarizer) technique or two crossed (or nearly crossed) polarizers. The images have been recorded with a CCD camera, digitized by a frame grabber with a resolution of at least $768 \times 576$ pixels and 24 bit color depth and saved for further processing/analysis.

Numerical simulations have aimed to compute the frequency dependent threshold voltage $U_{\mathrm{c}}(f)$ for the different EC patterns. The extended SM (with flexoelectricity included) has been used. Being interested in the onset behavior, linear stability analysis has been carried out as described in detail in [12]. Strong anchoring of the director and no slip condition for the flow have been assumed at the bounding plates, which have been ensured by a Galerkin method. The field variables have been expanded 
into sets of functions that vanish at the boundaries; the time periodicity has been guaranteed by Fourier expansion. Expansions in space and time have been truncated at the fourth and the seventh modes, respectively, which has been proved sufficient to obtain accuracy of better than $1 \%$ in $U_{\mathrm{c}}(f)$. This has been occasionally checked by increasing systematically the number of modes in space and time up to ten and fifteen, respectively, and monitoring the changes in $U_{\mathrm{c}}$. The growth rates $\mu(\mathbf{q}, U)$ for spatial fluctuations have been calculated as a function of the applied voltage. The minimum of the neutral surface $[\mu(\mathbf{q}, U)=0]$ provided the threshold voltage $U_{\mathrm{c}}$. The numerical calculations have been carried out with a Fortran code developed at the University of Bayreuth, using the same set of known material parameters (permittivities, conductivities, elastic moduli, viscosities), as for earlier calculations with the SM (for Phase 5 and Phase $5 \mathrm{~A}$ in [34], and for MBBA in [7, 35, 36]). The original measurements of these parameters have been reported in [34, 37, 38] for Phase 5 and in [39, 40, 41, 42, 43, 44] for MBBA.

The splay and bend flexoelectric coefficients, $e_{1}$ and $e_{3}$, should also be known, however, they are not easily measurable. Though various measuring techniques have been developed during the past decades, there is still a big controversy about the value and the sign of $e_{1}$ and $e_{3}$. The data obtained by different methods vary considerably even for the most studied nematic material, MBBA: $e_{1}+e_{3}$ ranges from $-2.3 \mathrm{pC} / \mathrm{m} 45$ to $-(54 \pm 10) \mathrm{pC} / \mathrm{m}$ 47], and $e_{1}-e_{3}$ from $(3.3 \pm 0.7) \mathrm{pC} / \mathrm{m}[46]$ to $(14 \pm 1) \mathrm{pC} / \mathrm{m}$ [47]. Up to now, only the sign of the sum of flexoelectric coefficients seems to be above dispute for MBBA: it appears to be negative $\left(e_{1}+e_{3}<0\right)$. For the other four compounds used in our experiments no data on the flexoelectric coefficients are at all currently available. It seems though, based on measurements on a number of (other) nematics, that at least the order of magnitude of $\sim 10 \mathrm{pC} / \mathrm{m}$ is established as a customary value for both $e_{1}$ and $e_{3}$ 48. As a consequence, during the numerical simulations we have regarded $e_{1}$ and $e_{3}$ as adjustable parameters, to be chosen to provide the best fit with the experiments.

\section{CONDUCTIVE S-EC AT LOW FREQUENCIES AND DIELECTRIC S-EC AT HIGH FREQUENCIES}

We start the discussion of the experimental results with systems exhibiting s-EC, where by choosing $d$ and $\sigma_{\perp}$ appropriately, the frequency range of the ac field covers $1 / \tau_{\mathrm{d}}$ and in most cases $1 / \tau_{\mathrm{q}}$ as well.

The first case we show is electroconvection in Phase 5 with $d=(3.4 \pm 0.5) \mu \mathrm{m}$ and $\sigma_{\perp}=8.2 \times 10^{-8}(\Omega \mathrm{m})^{-1}$. In this case $1 / \tau_{\mathrm{d}} \approx 60 \mathrm{~Hz}, 1 / \tau_{\mathrm{q}} \approx 1800 \mathrm{~Hz}$, and $1 / \tau_{\mathrm{v}} \approx$ $2 \times 10^{6} \mathrm{~Hz}$.

Threshold measurements are presented in Fig. 1. Below $f_{\mathrm{c}} \approx 550 \mathrm{~Hz}$ conductive s-EC (open circles) has been detected at onset in the form of traveling oblique rolls and traveling normal rolls. Above $f_{\mathrm{c}}$ traveling dielectric s-EC (bullets) has been observed.

A novel feature of the $U_{\mathrm{c}}(f)$ curve has been detected in the low frequency range, $20 \mathrm{~Hz} \lesssim f \lesssim 60 \mathrm{~Hz}$, where the threshold strongly decreases by lowering the frequency, in contrast to thick $(d \geq 10 \mu \mathrm{m})$ cells where $U_{\mathrm{c}}$ remains almost constant in the same frequency range.

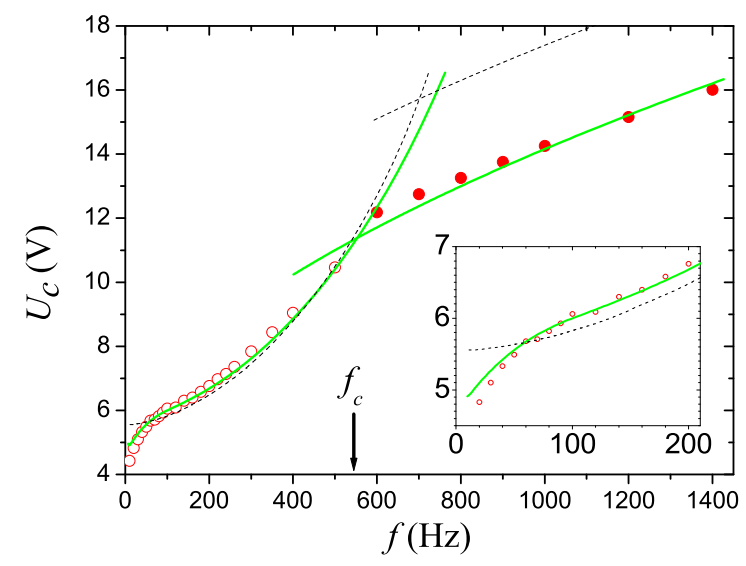

FIG. 1: (Color online) Frequency dependence of the s-EC threshold voltage $U_{\mathrm{c}}$ measured in Phase 5 with $d=(3.4 \pm$ $0.5) \mu \mathrm{m}$ - conductive s-EC (circles) and dielectric s-EC (bullets). The solid and the dashed lines are the results of the numerical analysis with and without the flexoelectric effect, respectively. The inset is the magnification of the low frequency range. The arrow indicates the crossover frequency $f_{\mathrm{c}}$.

Numerical calculations have been performed with the Phase 5 parameter set (see Table I), using the flexoelectric coefficients $e_{1}$ and $e_{3}$ as fitting parameters. In Fig. 1 the results are plotted both without the flexoelectric effect $\left(e_{1}=e_{3}=0\right.$, dashed lines, representing mode I and mode III) and with flexoelectric coefficients of $e_{1}=-26.5 \mathrm{pC} / \mathrm{m}$ and $e_{3}=-23.6 \mathrm{pC} / \mathrm{m}$ (solid lines, mode I+IV and mode II+III). For the conductive s-EC, the calculation without the flexoelectric effect does not show the bending down at low frequencies while with the $e_{1}$ and $e_{3}$ values given above a good match with the experimental data has been obtained (see the two curves in the inset of Fig. 1).

Calculations have been performed for a $d=20 \mu \mathrm{m}$ cell as well, using the same parameters from Table 1 (providing $1 / \tau_{\mathrm{d}} \approx 2 \mathrm{~Hz}, 1 / \tau_{\mathrm{q}} \approx 1800 \mathrm{~Hz}$, and $\left.1 / \tau_{\mathrm{v}} \approx 70 \times 10^{3} \mathrm{~Hz}\right)$. The results are plotted in Fig. 2. One can immediately see that the resulting $U_{\mathrm{c}}(f)$ for $e_{1}=e_{3}=0$ (mode I) nearly coincides with the one incorporating the flexoelectric effect (mode I+IV) almost in the whole conductive frequency range, in accordance with earlier conclusions [19]. Nevertheless a closer look at the curves (see the inset of Fig. 2 with a blow up of the plot) shows that 
TABLE I: Material parameters of Phase 5 and MBBA at $T=30^{\circ} \mathrm{C}$. Phase 5 parameters have also been used in numerical calculations for Phase $5 \mathrm{~A}$.

\begin{tabular}{|c|c|c|c|}
\hline Parameter & Unit & Phase 5 & MBBA \\
\hline \hline$K_{11}$ & $10^{-12} \mathrm{~N}$ & $9.8[34]$ & $5.89[39]$ \\
$K_{22}$ & $10^{-12} \mathrm{~N}$ & $4.6[34]$ & $3.7[39]$ \\
$K_{33}$ & $10^{-12} \mathrm{~N}$ & $12.7[34]$ & $7.56[39]$ \\
\hline$\sigma_{a} / \sigma_{\perp}$ & - & $0.69[34]$ & $0.5[40]$ \\
\hline$\varepsilon_{\perp}$ & - & $5.25[34]$ & $5.18[40]$ \\
$\varepsilon_{a}$ & - & $-0.184[34,37]$ & $-0.48[40]$ \\
\hline$\alpha_{1}$ & $10^{-3} \mathrm{Ns} / \mathrm{m}^{2}$ & $-39[34]$ & $-14.1[43]$ \\
$\alpha_{2}$ & $10^{-3} \mathrm{Ns} / \mathrm{m}^{2}$ & $-109.3[38]$ & $-80.0[43]$ \\
$\alpha_{3}$ & $10^{-3} \mathrm{Ns} / \mathrm{m}^{2}$ & $1.5[38]$ & $-1.513[43]$ \\
$\alpha_{4}$ & $10^{-3} \mathrm{Ns} / \mathrm{m}^{2}$ & $56.3[38]$ & $64.4[43]$ \\
$\alpha_{5}$ & $10^{-3} \mathrm{Ns} / \mathrm{m}^{2}$ & $82.9[38]$ & $57.2[43]$ \\
$\alpha_{6}$ & $10^{-3} \mathrm{Ns} / \mathrm{m}^{2}$ & $-24.9[38]$ & $-24.4[43]$ \\
\hline$e_{1}$ & $\mathrm{pC} / \mathrm{m}$ & -26.5 (fit) & -14.5 (fit) \\
$e_{3}$ & $\mathrm{pC} / \mathrm{m}$ & -23.6 (fit) & -20.5 (fit) \\
\hline
\end{tabular}

switching on the flexoelectricity results in the bending down of the threshold here too, similarly to the case of low $d$, only the effect occurs here at much lower frequencies.

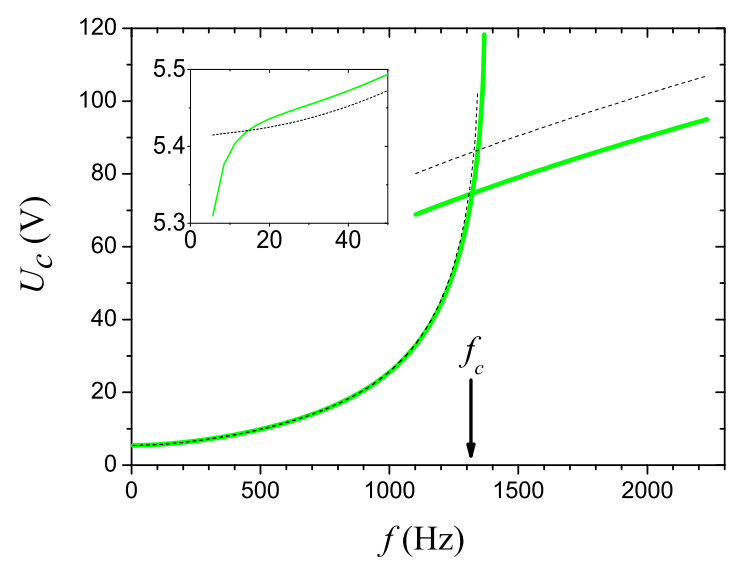

FIG. 2: (Color online) Numerical calculations for the frequency dependence of the s-EC threshold voltage $U_{\mathrm{c}}$ in a $d=20 \mu \mathrm{m}$ sample of Phase 5 with $\sigma_{\perp}=8.2 \times 10^{-8}(\Omega \mathrm{m})^{-1}$. Dashed lines are the conductive s-EC and the dielectric s-EC solutions with $e_{1}=e_{3}=0$, while the solid lines are the solutions with $e_{1}=-26.5 \mathrm{pC} / \mathrm{m}$ and $e_{3}=-23.6 \mathrm{pC} / \mathrm{m}$. The inset is the magnification of the low frequency range. The arrow indicates the crossover frequency $f_{\mathrm{c}}$.

The bending down of $U_{\mathrm{c}}$ at low $f$ when flexoelectricity is included can be understood from the earlier theoretical results [20] where it has been shown that flexoelectricity decreases the dc threshold voltage considerably (for MBBA the reduction is about 25\%). It could also be proved that in this dc limit none of the dimensionless equations of SM depend on $\sigma_{\perp} d^{2}$, hence the dc threshold is independent of $d$ and $\sigma_{\perp}$ [19]. In case of ac driving there is a wide frequency range (in the middle of the conductive regime) where flexoelectricity has much less influence on $U_{\mathrm{c}}$; it leaves the ac threshold almost unaltered, at least for not too thin cells. This means that at increasing $f$ the contribution from the flexoelectric charge separation (important at dc) fades away compared to that of the Coulomb charge density in a narrow frequency range at low $f$. The character of this transition from dc to ac driving has, however, not been studied rigorously either experimentally or theoretically so far.

In order to understand better the behavior of $U_{\mathrm{c}}$ we have carried out further simulations, carefully analyzing the low frequency range for a series of thicknesses when flexoelectricity is included. Results are shown in Fig. 3 for three thicknesses. The figure indicates that the transition from ac to dc (the bending down of $U_{\mathrm{c}}$ to its dc value) occurs for any thickness as expected; only the effect is compressed into a very narrow frequency range for large thicknesses. The corresponding values of $1 / \tau_{\mathrm{d}}$ are also indicated in Fig. 3 (circles). Their values fall into those frequency ranges (different for each $d$ ) in which the strong bending down of $U_{\mathrm{c}}$ occurs. We note, that according to its definition, $\tau_{\mathrm{d}}$ is the relaxation time of a homogeneously deformed director state (wavenumber $|\mathbf{q}|=0$ ). The relaxation time of the pattern, $1 / \tau_{\mathrm{p}}$, is shorter, because the growth/decay rate $\left(\mu=1 / \tau_{\mathrm{p}}\right)$ of a periodic $(|\mathbf{q}| \neq 0)$ distortion increases with $|\mathbf{q}|[49$, 50]. Therefore $1 / \tau_{\mathrm{p}}>1 / \tau_{\mathrm{d}}$ would be a more appropriate characteristic frequency of the system than $1 / \tau_{\mathrm{d}}$. A rigorous quantitative comparison of $1 / \tau_{\mathrm{p}}$ with $f$ is, however, problematic since $|\mathbf{q}|$ and hence $\tau_{\mathrm{p}}$ are frequency dependent.

The crossover between the regimes where the Coulomb charge separation or the flexoelectric charge separation dominates, is reflected in the curvature of the $U_{\mathrm{c}}(f)$ curves. It is convenient to use the location of the in- 


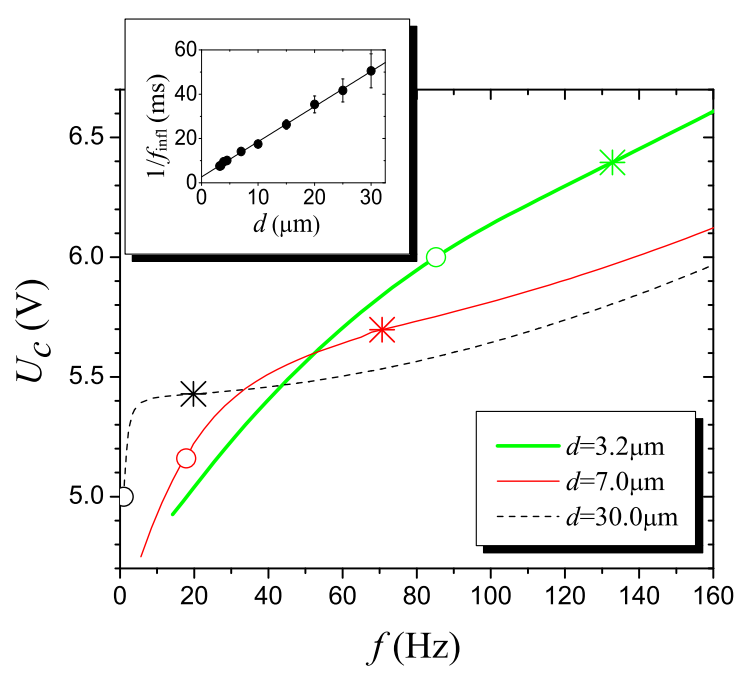

FIG. 3: (Color online) Numerically calculated frequency dependence of the s-EC threshold voltage $U_{\mathrm{c}}$ for samples of various thicknesses in Phase 5 with $\sigma_{\perp}=8.2 \times 10^{-8}(\Omega \mathrm{m})^{-1}$, $e_{1}=-26.5 \mathrm{pC} / \mathrm{m}$ and $e_{3}=-23.6 \mathrm{pC} / \mathrm{m}$. The circles and the star symbols on the curves denote $1 / \tau_{\mathrm{d}}$ and the inflection point at $f_{\text {infl }}$, respectively. Inset: the thickness dependence of $1 / f_{\text {infl. }}$.

flection point at $f_{\text {infl }}$ (where the curvature changes sign, indicated by stars in Fig. 3) as a quantitative measure. As one sees values of $f_{\text {inf }}$ are close to $1 / \tau_{\mathrm{d}}$, but interestingly, $1 / f_{\text {inf }}$ is linear with $d$ (see in the inset of Fig. 3), in contrast to the $d^{2}$ dependence of $\tau_{\mathrm{d}}$.

Finally, we mention that for the high frequency end of the conductive regime the influence of flexoelectricity becomes again noticeable though less dramatic. It considerably enhances the $d$-dependence (see in Fig. 3) which would be almost negligible at $e_{1}=e_{3}=0$, especially for higher thicknesses. Furthermore it shifts $f_{\mathrm{c}}$ to lower frequencies as seen in Fig. 1.

The shift of $f_{\mathrm{c}}$ is mainly due to the fact that in the dielectric regime of s-EC the flexoelectric effect decreases the threshold voltage drastically - compare the dashed curve $\left(e_{1}=e_{3}=0\right.$, mode III) and the solid line $\left(e_{1}=-26.5 \mathrm{pC} / \mathrm{m}\right.$ and $e_{3}=-23.6 \mathrm{pC} / \mathrm{m}$, mode II $\left.+\mathrm{III}\right)$ for the dielectric solutions above $f_{\mathrm{c}}$ in Figs. 1 1 and 2 The reduction reaches about $16 \%$ even in the relatively thick $(d=20 \mu \mathrm{m})$ sample, which agrees with recent findings for MBBA [32]. Consequently, flexoelectric effects cannot be neglected in case of dielectric s-EC even in the thick samples. We also mention here that no peculiar effect on $U_{\mathrm{c}}(f)$ is observed in the vicinity of $1 / \tau_{\mathrm{q}} \approx 1800 \mathrm{~Hz}$ (see Fig. 2).

Summarizing the results above one can conclude that with the above mentioned values of $\sigma_{\perp}, e_{1}$ and $e_{3}$, an excellent agreement has been found between the experimental results (symbols) and the numerical calculations (solid lines in Fig. 1) for Phase 5 in the whole experi- mentally covered frequency range. Note that the fitted $e_{1}$ and $e_{3}$ values are realistic in a sense that they are of the same order of magnitude as those measured for other nematics.

Similar results have also been obtained on Phase 5A and MBBA samples in which only the conductive regime of s-EC has been detected. Phase 5A has been investigated in a sample of $d=3.1 \mu \mathrm{m}$. At dc voltage as well as at very low frequencies (up to $f \approx 3 \mathrm{~Hz}$ ) static stripe patterns, the so called flexoelectric domains [17, 51] have been observed at a threshold $U_{\mathrm{f}}$. Above $f=3 \mathrm{~Hz}$ only conductive s-EC patterns have been seen: up to $f=40 \mathrm{~Hz}$ stationary oblique rolls and above $f=40 \mathrm{~Hz}$ traveling oblique rolls and traveling normal rolls. The measured frequency dependence of the threshold voltage is presented in Fig. 4 by open circles. Below $f \approx 50 \mathrm{~Hz}$ it appears that $U_{\mathrm{c}}$ decreases considerably as approaching $f=0$ (see the inset in Fig. (4), in a similar fashion as seen for the Phase 5 in Fig. 1) Again the bending down occurs in the frequency range of $1 / \tau_{\mathrm{d}}$.

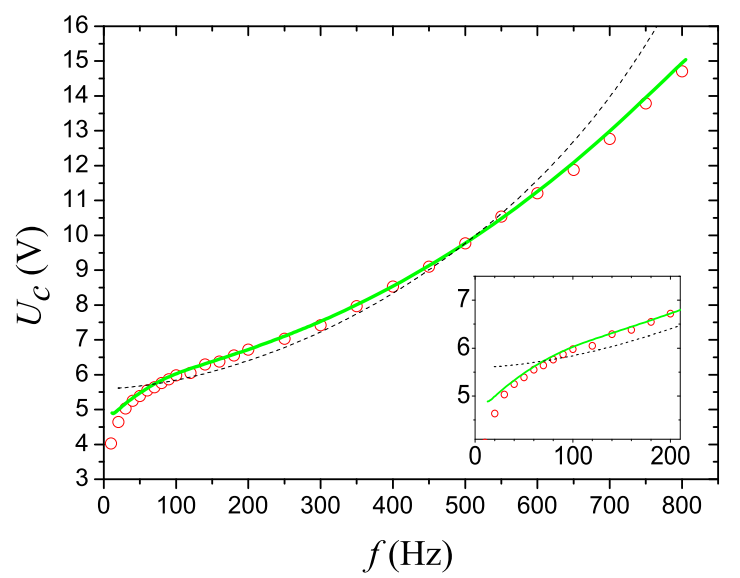

FIG. 4: (Color online) Frequency dependence of the threshold voltage $U_{\mathrm{c}}$ in the conductive regime for a sample of Phase $5 \mathrm{~A}$ with $d=3.1 \mu \mathrm{m}$. Circles stand for experimental data. The solid line represents the $U_{\mathrm{c}}(f)$ curve calculated numerically with Phase 5 parameters (same as in Figure 1, with nonzero $e_{1}$ and $\left.e_{3}\right)$ and with $\sigma_{\perp}=9.2 \times 10^{-8}(\Omega \mathrm{m})^{-1}$. The dashed line is the calculated solution for $e_{1}=e_{3}=0$. The inset is the magnification of the low frequency range.

Phase 5A has the same chemical composition as Phase 5 except that Phase $5 \mathrm{~A}$ is doped with some ionic salt in order to increase the electric conductivity. Therefore, in the numerical calculations for Phase $5 \mathrm{~A}$ we have used the material parameters of Phase 5 (given in Table 【) with $\sigma_{\perp}=9.2 \times 10^{-8}(\Omega \mathrm{m})^{-1}$, and the flexoelectric coefficients $e_{1}=-26.5 \mathrm{pC} / \mathrm{m}$ and $e_{3}=-23.6 \mathrm{pC} / \mathrm{m}$ that have been obtained from the best fit in Fig. 11. The calculated threshold curve for conductive s-EC (solid line in Fig. 4) agrees very well with the experimental data in the whole frequency range. 
For reference, in Fig. 4 we have also plotted the conductive s-EC threshold $U_{\mathrm{c}}(f)$ without the flexoelectric effect (i.e., for $e_{1}=e_{3}=0$; dashed line). The same conclusions can be drawn here as those for Phase 5: the $U_{\mathrm{c}}(f)$ curve with $e_{1}=e_{3}=0$ deviates significantly and systematically from that with nonzero $e_{1}$ and $e_{3}$ (and also from the experimental data), especially in the low frequency range (below $f \approx 50 \mathrm{~Hz}$ ) and at high frequencies (above $600 \mathrm{~Hz})$.

Up to now the most studied nematic EC material is presumably MBBA which we have investigated too, in a cell of $d=3.2 \mu \mathrm{m}$. At dc voltage neither flexoelectric domains, nor periodic EC patterns have been detected up to $U=80 \mathrm{~V}$. However, at and above $f=0.1 \mathrm{~Hz}$ a conductive s-EC pattern has emerged. Frequency dependence of the threshold voltage measured in this thin layer of MBBA is shown in Fig. 5 (circles). Again, below $f \approx 30 \mathrm{~Hz} U_{\mathrm{c}}(f)$ bends down considerably while approaching $f \rightarrow 0$, in a similar fashion as seen for Phase 5 and Phase $5 \mathrm{~A}$.

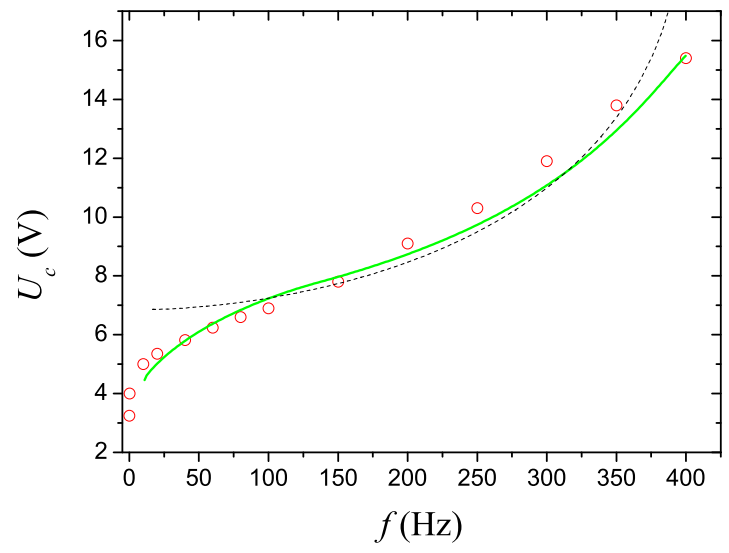

FIG. 5: Frequency dependence of the conductive s-EC threshold voltage $U_{\mathrm{c}}$ measured on a sample of MBBA with $d=$ $3.2 \mu \mathrm{m}$ (circles). The solid line represents the $U_{\mathrm{c}}(f)$ curve calculated numerically with MBBA parameters from Table I and with $\sigma_{\perp}=9 \times 10^{-8}(\Omega \mathrm{m})^{-1}, e_{1}=-14.5 \mathrm{pC} / \mathrm{m}$ and $e_{3}=-20.5 \mathrm{pC} / \mathrm{m}$. The dashed line is the solution for the same parameter set, but with $e_{1}=e_{3}=0$.

The MBBA parameter set from Table $\prod$ has been used in the numerical calculations. The solid line in Fig. 5 obtained by the simulations for $e_{1}=-14.5 \mathrm{pC} / \mathrm{m}, e_{3}=$ $-20.5 \mathrm{pC} / \mathrm{m}$ and $\sigma_{\perp}=9 \times 10^{-8}(\Omega \mathrm{m})^{-1}$ represents a fairly good match with the experimental data, in contrast to the curve with $e_{1}=e_{3}=0$ (dashed line in Fig. 5) which deviates significantly. One has to note here that the fitted values of $e_{1}$ and $e_{3}$ fall in the mid-range of measured values for MBBA [48], and in particular agree reasonably well with the values $\left|e_{1}\right|=15 \mathrm{pC} / \mathrm{m}$ and $\left|e_{3}\right|=30 \mathrm{pC} / \mathrm{m}$ reported in [52, 53].

\section{DIELECTRIC S-EC AT LOW FREQUENCIES}

With a careful preparation procedure we were able to produce thin $(d=3.4 \mu \mathrm{m})$ Phase 5 samples with a conductivity as low as about $\sigma_{\perp} \approx 1 \times 10^{-8}(\Omega \mathrm{m})^{-1}$. These samples exhibited convection-free flexoelectric domains at a threshold $U_{\mathrm{f}}$ at dc and at very low frequency (up to $f=7.5 \mathrm{~Hz}$ ) ac driving, similarly to the situation in Phase $5 \mathrm{~A}$. Above $f=7.5 \mathrm{~Hz}$, in a narrow frequency range (up to $f \approx 20 \mathrm{~Hz}$ ) flexoelectric domains and traveling dielectric s-EC oblique rolls coexist with slightly different thresholds. At higher frequencies dielectric s-EC could be detected in the form of traveling oblique, or traveling normal rolls.

The frequency dependence of $U_{\mathrm{c}}$ in this sample (depicted with bullets in Fig. 6) is, however, rather unusual and surprising, considering that both the SM and previous experiments on dielectric s-EC provided a square root-like $U_{\mathrm{c}}(f)$ function. Instead, here $U_{\mathrm{c}}(f)$ bends up as $f \rightarrow 0$, i.e. it is a non-monotonic function with an expressed minimum at $f_{\min }$ in the frequency range of $1 / \tau_{\mathrm{d}}$. Moreover, at higher frequencies (above the minimum) $U_{\mathrm{c}}(f)$ is linear within the experimental error. Performing numerical simulations with the Phase 5 parameter set in Table 1 and with $\sigma_{\perp}=1 \times 10^{-8}(\Omega \mathrm{m})^{-1}$, we have found that the calculated dielectric threshold curve $U_{\mathrm{c}}(f)$ (shown as the solid line with the lowest $U_{\mathrm{c}}$ in Fig. 6) is monotonic, has a smaller slope (above $60 \mathrm{~Hz}$ ) and provides lower thresholds (especially at high $f$ ) than found experimentally.

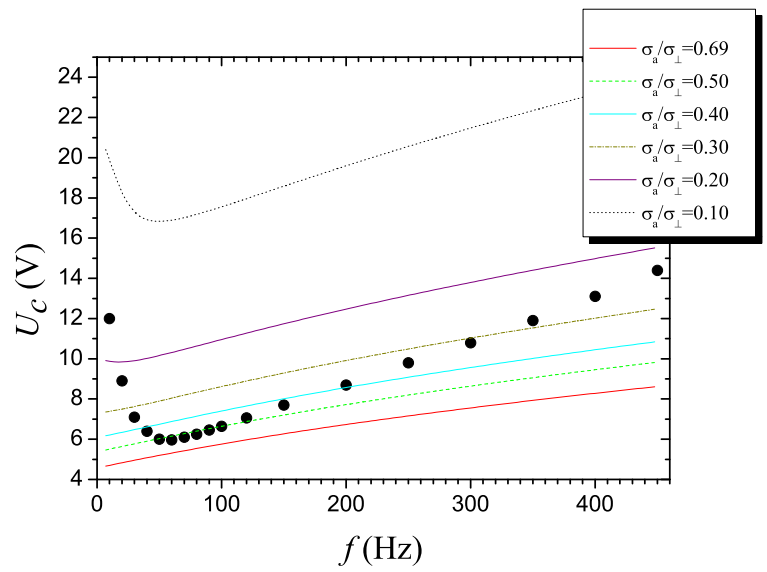

FIG. 6: (Color online) Frequency dependence of the dielectric threshold voltage $U_{\mathrm{c}}$ measured in a $d=3.4 \mu \mathrm{m}$ sample of Phase 5 (bullets). Lines represent results of numerical calculations for Phase 5 with $e_{1}=-26.5 \mathrm{pC} / \mathrm{m}, e_{3}=-23.6 \mathrm{pC} / \mathrm{m}$, and $\sigma_{\perp}=1 \times 10^{-8}(\Omega \mathrm{m})^{-1}$ for a series of $\sigma_{a} / \sigma_{\perp}$ values.

In order to test whether the extended SM is able at all to provide an $U_{\mathrm{c}}(f)$ dependence with at least a qualitative similarity to the measured data, we have performed 
simulation experiments. First we have checked the influence of the conductivity systematically in a wide range of $10^{-12}(\Omega \mathrm{m})^{-1} \leq \sigma_{\perp} \leq 10^{-8}(\Omega \mathrm{m})^{-1}$, where only dielectric s-EC is expected. We could verify that the calculated $U_{\mathrm{c}}(f)$ remains monotonic in this $\sigma_{\perp}$ range, and $\mathrm{d} U_{\mathrm{c}} / \mathrm{d} f$ is not affected significantly. Consequently, the novel frequency dependence of the threshold (bending up) cannot be interpreted by a pure reduction of the conductivity.

We have also varied the anisotropy of the conductivity motivated by recent numerical results 12 indicating that a decrease of $\sigma_{a} / \sigma_{\perp}$ increases the slope $\mathrm{d} U_{\mathrm{c}} / \mathrm{d} f$ and tends to make $U_{\mathrm{c}}(f)$ linear. In Fig. 6] we present the calculated threshold curves for five additional $\sigma_{a} / \sigma_{\perp}$ values, leaving all other parameters in Table @ unaltered. It is seen that $U_{\mathrm{c}}(f)$ as well as $\mathrm{d} U_{\mathrm{c}} / \mathrm{d} f$ shift to higher values with the decrease of $\sigma_{a} / \sigma_{\perp}$, moreover, $U_{\mathrm{c}}(f)$ becomes non-monotonic for $\sigma_{a} / \sigma_{\perp} \leq 0.2$ with a bending up of $U_{\mathrm{c}}(f)$ at low frequencies. Note that the curve calculated for $\sigma_{a} / \sigma_{\perp}=0.1$ reproduces qualitatively all features of the experimental $U_{\mathrm{c}}(f)$, except that it is shifted to higher voltages.

To demonstrate the role of flexoelectricity in the novel frequency dependence of the dielectric regime, in Fig. 7 we have plotted $U_{\mathrm{c}}(f)$ calculated with $\sigma_{a} / \sigma_{\perp}=0.1$ both for mode III (i.e., without flexoelectricity, dashed line) and for mode II+III (i.e., with flexoelectricity, solid line). Obviously, when flexoelectric effects are neglected, the threshold is even higher, $U_{\mathrm{c}}(f)$ decreases monotonously when $f \rightarrow 0$ and the bending up is not reproduced, so there is no agreement with the experimental data even on a qualitative level. On the other hand, if one takes into account the flexoelectric coefficients given in Table @ an acceptable qualitative agreement is achieved: the curve has a minimum at about the same $f_{\text {min }}$ frequency where the experimental data, and for $f>f_{\text {min }}$ the steepness $\mathrm{d} U_{\mathrm{c}} / \mathrm{d} f$ is roughly the same as that in the experiments. Therefore, the bending up can undoubtedly be attributed to flexoelectricity, and $f_{\min }$ is a natural indicator for the frequency range where the crossover between dominantly flexoelectric and dominantly Coulomb charge separation mechanisms occur.

Finally we mention that (similarly to the case discussed for Fig. 2) no peculiar effect of flexoelectricity on $U_{\mathrm{c}}(f)$ is observed in the vicinity of $1 / \tau_{\mathrm{q}} \approx 220 \mathrm{~Hz}$ (see Figs. 6] and (7).

Systematic numerical calculations at $\sigma_{a} / \sigma_{\perp}=0.1$ have shown that while keeping $\sigma_{\perp}$ (and thus $\tau_{\mathrm{q}}$ ) constant, $f_{\min }$ shifts to higher $f$ with the decrease of $d$ (i.e., of $\tau_{\mathrm{d}}$ ) see Fig. 8. Despite of the fact that $1 / f_{\text {min }}$ increases linearly with $d$ up to $d=8 \mu \mathrm{m}$ (similarly to $1 / f_{\text {infl }}$ of conductive s-EC), one finds the values of $1 / f_{\min }(d)$ close to $\tau_{\mathrm{d}}(d)$, and the best fit $1 / f_{\min } \propto d^{2}$ does not differ significantly from $\tau_{\mathrm{d}}(d)$ (see inset of Fig. 8). At $d=20 \mu \mathrm{m}$ $U_{\mathrm{c}}(f)$ becomes a monotonically increasing function (at least above $f=5 \mathrm{~Hz}$ ). One has to mention here that in our calculations we have not resolved the very low frequency range $(\sim 1 \mathrm{~Hz})$, where the theoretical analysis becomes more difficult due to numerical problems and the

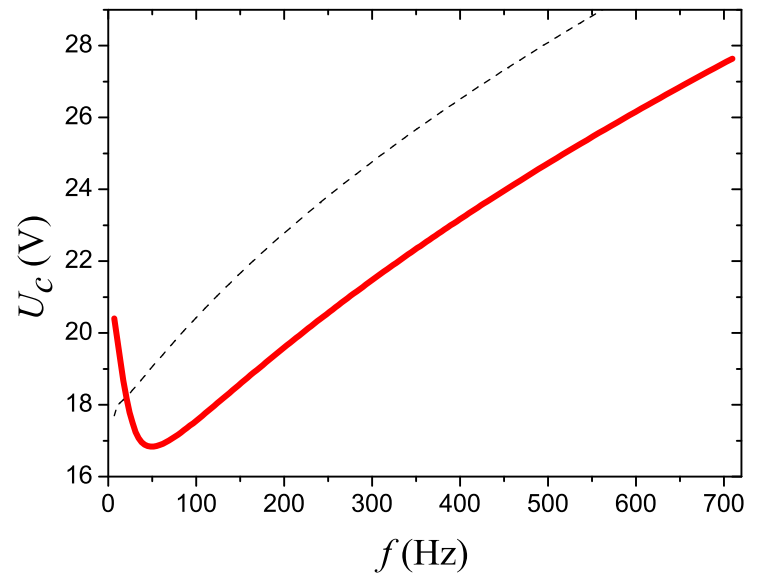

FIG. 7: (Color online) Numerical calculations of the threshold $U_{\mathrm{c}}(f)$ for Phase 5 with $\sigma_{a} / \sigma_{\perp}=0.1, \sigma_{\perp}=1 \times 10^{-8}(\Omega \mathrm{m})^{-1}$, and $d=3.4 \mu \mathrm{m}$ : dashed line is dielectric s-EC with $e_{1}=e_{3}=$ 0 ; solid line is the dielectric mode with $e_{1}=-26.5 \mathrm{pC} / \mathrm{m}$, $e_{3}=-23.6 \mathrm{pC} / \mathrm{m}$.

experiments also require special care (and are, therefore, typically avoided). From Fig. 8 one also sees that the steepness of $U_{\mathrm{c}}(f)$ in the high frequency range (where the function is close to linear) also depends on $d ; \mathrm{d} U_{\mathrm{c}} / \mathrm{d} f$ increases with $d$.

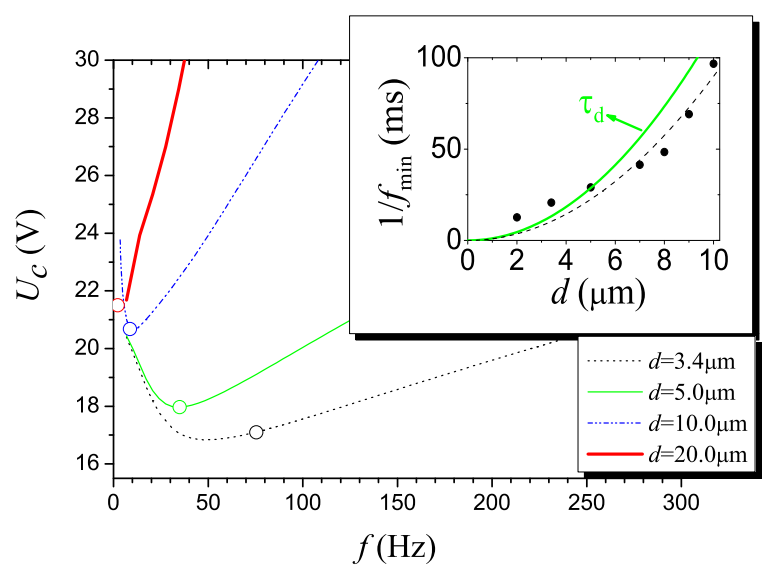

FIG. 8: (Color online) Frequency dependence of the dielectric s-EC threshold, calculated with Phase 5 parameters for $\sigma_{a} / \sigma_{\perp}=0.1$ and with $e_{1}=-26.5 \mathrm{pC} / \mathrm{m}, e_{3}=-23.6 \mathrm{pC} / \mathrm{m}$, $\sigma_{\perp}=1 \times 10^{-8}(\Omega \mathrm{m})^{-1}$ for different sample thicknesses $d$. Circles denote $1 / \tau_{\mathrm{d}}$ for each $d$. In the inset the thickness dependence of $1 / f_{\min }$ (bullets) and that of $\tau_{\mathrm{d}}$ (solid line) is presented together with the best fit $1 / f_{\min } \propto d^{2}$ (dashed line).

The other situation is when $\tau_{\mathrm{q}}$ is tuned via $\sigma_{\perp}$ while keeping $d$ and thus $\tau_{\mathrm{d}}$ constant $\left(1 / \tau_{\mathrm{d}}=75.5 \mathrm{~Hz}\right)$. The influence of $\sigma_{\perp}$ on $U_{\mathrm{c}}(f)$ is illustrated in Fig. 9] With 
the increase of the conductivity (i.e, of $1 / \tau_{\mathrm{q}}$ ), both $f_{\text {min }}$ and the lowest threshold value $U_{\mathrm{c}}\left(f_{\min }\right)$ increase.

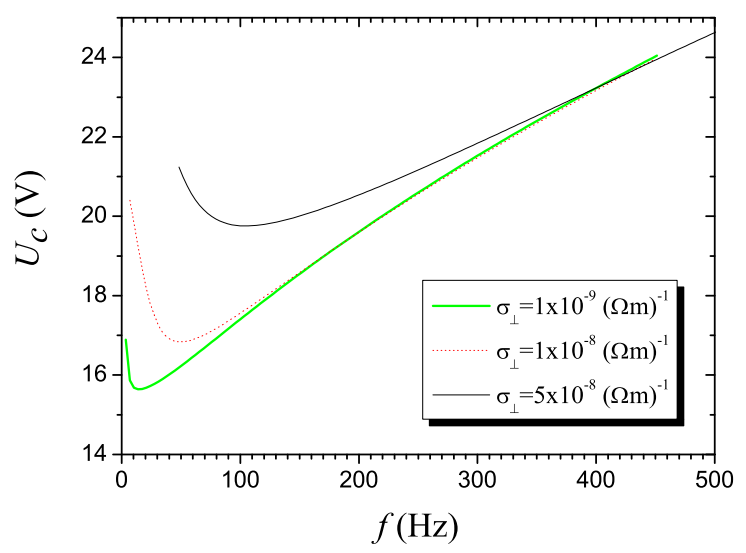

FIG. 9: (Color online) Results of numerical calculations for Phase 5 with $\sigma_{a} / \sigma_{\perp}=0.1, e_{1}=-26.5 \mathrm{pC} / \mathrm{m}, e_{3}=$ $-23.6 \mathrm{pC} / \mathrm{m}$ and $d=3.4 \mu \mathrm{m}$ for three different conductivities $\sigma_{\perp}$.

Samples of the nematic Phase 4 have also been investigated at $T=30^{\circ} \mathrm{C}$. Measurements on the frequency dependence of $U_{\mathrm{c}}$ are shown in Fig. 10 for a $d=3.2 \mu \mathrm{m}$ cell (circles). At dc voltage (solid triangle) and at low frequencies (up to $f=15 \mathrm{~Hz}$ ) flexoelectric domains [see in Fig. 11(a)] have been observed with a tendency of $U_{f}$ increasing with the frequency. At $f=15 \mathrm{~Hz}$ the thresholds for flexoelectric domains and for traveling dielectric oblique rolls almost coincide (see the coexisting patterns in Fig. 11(b) - a similar behavior has also been reported in [54]). At higher $f$ flexoelectric domains could not be detected. Instead, traveling dielectric oblique rolls [Fig. [11(c)] or traveling dielectric normal rolls have been observed. The threshold curve for these traveling rolls has a minimum similarly to that observed in dielectric s-EC of Phase 5. In contrast to the thin cell, however, experimental data obtained on a $d=12.4 \mu \mathrm{m}$ thick sample of Phase 4 (star symbols in Fig. 10 and in its inset) demonstrate a monotonically increasing $U_{\mathrm{c}}(f)$ (at least for $f \geq 10 \mathrm{~Hz}$ ). At the same time, Fig. 10 represents an experimental support of numerical results shown in Fig. 8 obtained though with Phase 5 parameters (unfortunately, in Phase 5 samples with $d \geq 10 \mu \mathrm{m}$, dielectric s-EC could not be obtained at low enough $f$ ).

\section{NONSTANDARD EC}

Previous measurements have shown that in samples of $8 / 7$ with typical thickness $(d \geq 10 \mu \mathrm{m})$, ns-EC takes place in the whole investigated frequency range at $T \leq$ $85^{\circ} \mathrm{C}$, characterized by a linear $U_{\mathrm{c}}(f)$ dependence. In the temperature range $85^{\circ} \mathrm{C}<T<90^{\circ} \mathrm{C}$ both ns-EC and s-

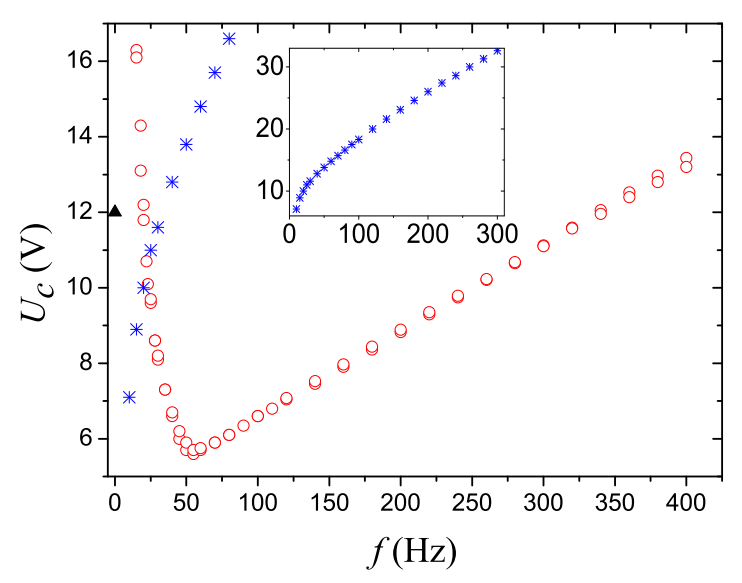

FIG. 10: (Color online) Frequency dependence of the s-EC threshold voltage $U_{\mathrm{c}}$ measured in a sample of Phase 4 with $d=3.2 \mu \mathrm{m}$ (circles). At dc voltage, the threshold for the flexoelectric domains is also indicated (solid triangle). For comparison, experimental data obtained on another sample of Phase 4 with $d=12.4 \mu \mathrm{m}$ is also plotted (stars). The inset displays experimental results on the $d=12.4 \mu \mathrm{m}$ cell in the whole frequency range studied.
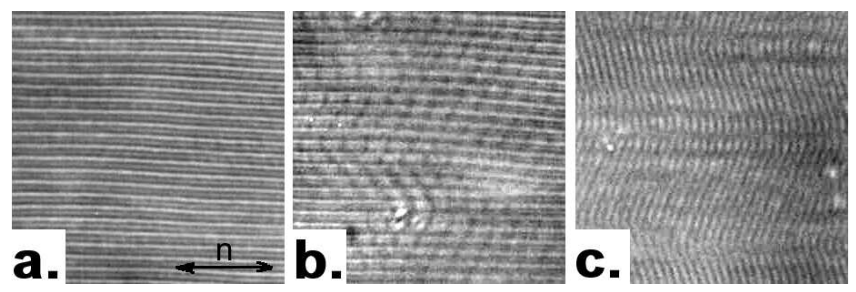

FIG. 11: Snapshots of patterns taken slightly above the threshold in a $d=3.2 \mu \mathrm{m}$ sample of Phase 4 . (a.) flexoelectric domains at dc voltage; (b.) coexisting flexoelectric domains and traveling dielectric oblique rolls at $f=15 \mathrm{~Hz}$; (c.) traveling dielectric oblique rolls at $f=70 \mathrm{~Hz}$. The arrow denotes the initial director. The physical size of the images is $(65 \times 65) \mu \mathrm{m}^{2}$.

EC could be observed depending on $f$ and $d$, while above $T=90^{\circ} \mathrm{C}$ always s-EC has appeared at onset [26].

In accordance with the above experimental findings, in our $d=3.4 \mu \mathrm{m}$ sample of $\mathbf{8} / \mathbf{7} \mathrm{ns}-\mathrm{EC}$ has been found below $T \approx 90^{\circ} \mathrm{C}$. At higher frequencies, similarly to thick cells, a linear frequency dependence of the threshold voltage has been detected which is illustrated in Fig. 12 for three different temperatures. However, at lower $f$ the frequency dependence of $U_{\mathrm{c}}$ is different from the one observed in thicker samples [26]. The threshold voltage increases abruptly here when $f \rightarrow 0$ as shown in Fig. 12 i.e., $U_{\mathrm{c}}(f)$ becomes a non-monotonic function with a minimum $U_{\text {cmin }}$ at $f_{\text {min }}$, similarly to the case of dielectric s-EC in Phase 5 and Phase 4 samples.

Studies on the temperature dependence of $U_{\mathrm{c}}(f)$ in the 


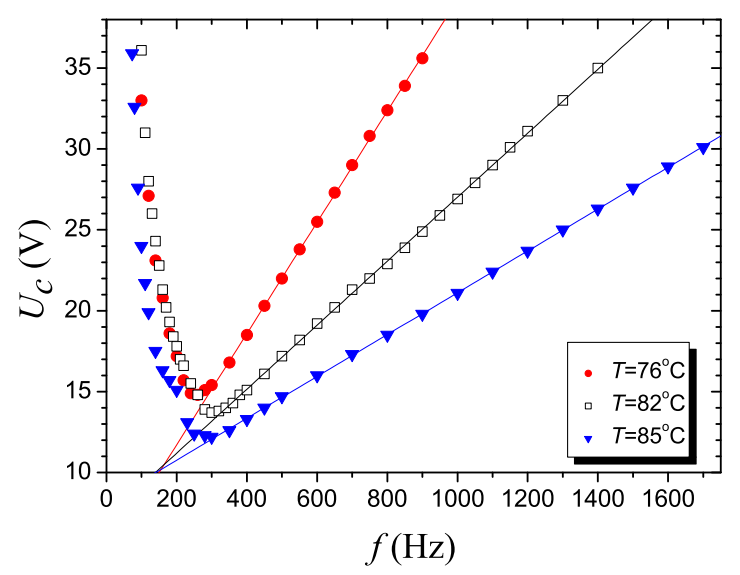

FIG. 12: (Color online) Frequency dependence of the ns-EC threshold voltage $U_{\mathrm{c}}$ measured in a sample of $8 / 7$ with $d=$ $3.4 \mu \mathrm{m}$ for three different temperatures $T$. Solid lines represent linear fit to data at high frequencies (above the minima of the curves).

sample of $\mathbf{8 / 7}$ with $d=3.4 \mu \mathrm{m}$ have revealed that: (i.) $U_{\text {cmin }}$ reduces with the increase of $T$; (ii.) $f_{\min }$ increases with $T$; (iii.) the steepness of $U_{\mathrm{c}}(f)$ in the high frequency range (where the function is linear) is also temperature dependent; $\mathrm{d} U_{\mathrm{c}} / \mathrm{d} f$ diminishes linearly with the increase of $T$.

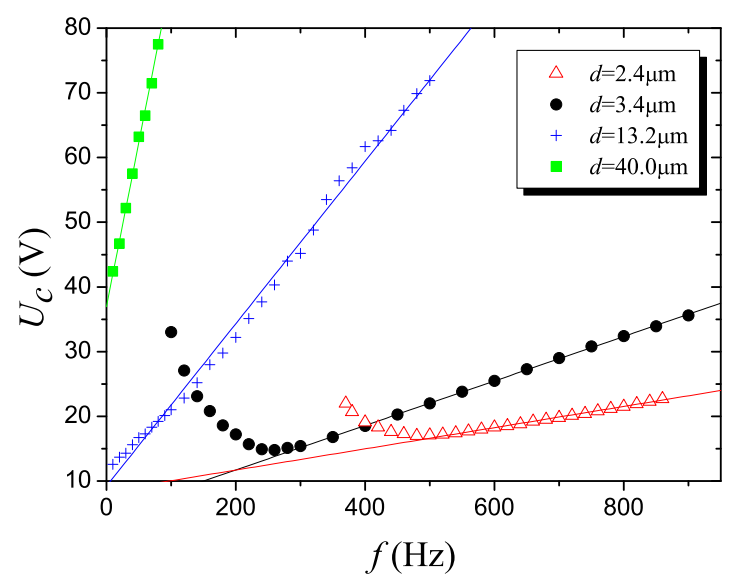

FIG. 13: (Color online) ns-EC threshold curves $U_{\mathrm{c}}(f)$ measured in samples of $8 / 7$ of different thicknesses $d$ at $T=76^{\circ} \mathrm{C}$. Solid lines represent linear fit to data.

In an even thinner $(d=2.4 \mu \mathrm{m})$ sample of $8 / 7$ no periodic EC structure could be observed, instead, a largescale, non-periodic pattern has appeared at a critical voltage $U_{\mathrm{ls}}$. Since a similar non-periodic, large-scale pattern has also been observed as a concomitant phe- nomenon of the ns-EC pattern at $U_{\mathrm{c}}$ in thicker samples, in Fig. 13 we compare this $U_{\mathrm{ls}}$ with $U_{\mathrm{c}}$ of ns-EC measured in samples of different thicknesses at $T=76^{\circ} \mathrm{C}$. It is seen that the novel non-monotonic behavior is restricted to the two smallest thicknesses $(d=2.4 \mu \mathrm{m}$ and $d=3.4 \mu \mathrm{m}$ ). $f_{\min }$ increases as $d$ is lowered (it is about $220 \mathrm{~Hz}$ for the $d=3.4 \mu \mathrm{m}$ sample, and is roughly $500 \mathrm{~Hz}$ for $d=2.4 \mu \mathrm{m})$. Therefore, one can conclude that $f_{\min }$ depends stronger on $d$, than on the material parameters (compare with the much weaker temperature dependence of this minimum in Fig. 12). It can be noted that while $U_{\text {cmin }}$ and $f_{\text {min }}$ grow, $\mathrm{d} U_{\mathrm{c}} / \mathrm{d} f$ decreases with lowering the sample thickness $d$.

The temperature range where ns-EC is observable extends to higher $T$ in thin samples compared to thicker ones. To demonstrate this, we plotted in Fig. 14 the frequency dependence of the threshold voltage at $T \approx 90^{\circ} \mathrm{C}$ for three different sample thicknesses. In the thick, $d=$ $40 \mu \mathrm{m}$, cell s-EC exists in the whole accessible frequency range. At an intermediate thickness of $13.2 \mu \mathrm{m}$ a s-EC to ns-EC transition has been observed at $f \approx 500 \mathrm{~Hz}$. In the thin, $d=3.4 \mu \mathrm{m}$, cell ns-EC has been detected in the whole frequency range. In this latter case a nonmonotonic $U_{\mathrm{c}}(f)$ is observed, just as at lower temperatures, or in the dielectric s-EC of Phase 5 and Phase 4. Fig. 14demonstrates that a s-EC to ns-EC transition can also be induced by reducing the sample thickness (while keeping the temperature and frequency fixed), in addition to the already reported temperature, frequency and electric field induced transitions [26].

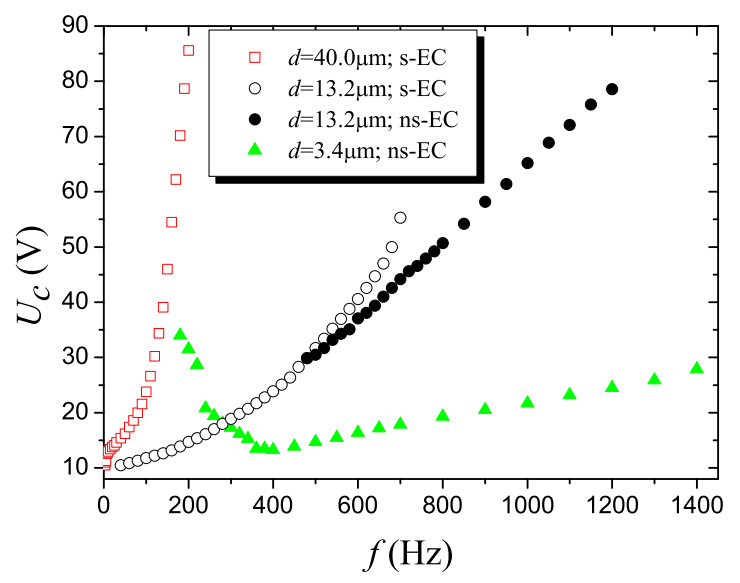

FIG. 14: Frequency dependence of the threshold voltage $U_{\mathrm{c}}$ measured in $8 / 7$ samples of different thickness $(d=3.4 \mu \mathrm{m}$, $13.2 \mu \mathrm{m}$ and $40 \mu \mathrm{m}$ ) at $T \approx 90^{\circ} \mathrm{C}$ (in the vicinity of the s-EC to ns-EC transition). Open symbols denote the s-EC threshold, while closed symbols stand for ns-EC.

Recent theoretical advances (the analysis of the extended SM) have shown that the flexoelectric charge separation mechanism could be responsible for the occurrence of ns-EC [12]. Numerical simulations based on 
the extended SM could actually yield a good agreement with experimental results on a thick sample of the compound 8/7 using a reasonable guess for unknown material parameters. Extension of the numerical analysis to smaller thicknesses and lower frequencies is, however, not so straightforward in the parameter range of ns-EC as for s-EC. In addition some important parameters (e.g., viscosities and flexoelectric coefficients) should first be determined by independent measurements. As a consequence a theoretical $U_{\mathrm{c}}(f)$ dependence for Fig. 14] could not yet been provided; it remains a target for further studies.

\section{DISCUSSION}

We have reported about measurements of the threshold voltage of electroconvection patterns in various nematic liquid crystals focusing mainly on thin $(d \approx 3.1-3.5 \mu \mathrm{m})$ samples in which $1 / \tau_{\mathrm{d}}$ is relatively large. These samples have exhibited patterns of various types in the vicinity of $1 / \tau_{\mathrm{d}}$ : conductive s-EC, dielectric s-EC as well as nonstandard EC. For all compounds and pattern types we have found a novel frequency dependence of the threshold voltage at low frequencies, which deviates significantly from that typically seen in thicker cells with smaller $1 / \tau_{\mathrm{d}}$. This indicates that this novel phenomenon is quite robust. The character of the novel $U_{\mathrm{c}}(f)$ behavior seems, however, to depend on the type of the pattern. On the one hand, whenever conductive s-EC developes at onset, $U_{\mathrm{c}}(f)$ expresses a pronounced decrease (bending down) if the frequency is lowered, thus $U_{\mathrm{c}}(f)$ has an inflection point. On the other hand, if the onset pattern is dielectric s-EC in the vicinity of $1 / \tau_{\mathrm{d}}, U_{\mathrm{c}}(f)$ exhibits an abrupt increase (bending up) towards lower $f$, so $U_{\mathrm{c}}(f)$ has a minimum. In case of ns-EC, the low frequency behavior is similar to that of the dielectric s-EC. As according to recent theoretical results the ns-EC is actually a dielectric mode [12], one can conclude that whether $U_{\mathrm{c}}(f)$ bends down or up at $f \rightarrow 0$, depends on the spatiotemporal symmetry of the solution at onset. The significant changes in the curvature of $U_{\mathrm{c}}(f)$ occur in the frequency range of $1 / \tau_{\mathrm{d}}$, even though some characteristic frequencies $\left(f_{\text {infl }}\right.$ for the conductive or $f_{\text {min }}$ for the dielectric pattern) do not scale precisely with $\tau_{\mathrm{d}}$.

Besides the experiments, the threshold voltages have also been calculated numerically for s-EC in a linear stability analysis using both the standard model of electroconvection (neglecting flexoelectricity), and the extended SM (including the flexoelectric effect). The simulations have shown that in the absence of flexoelectricity thin as well as thick cells exhibit the "regular" $U_{\mathrm{c}}(f)$ behavior, without the novel dependence at low $f$. Including, however, flexoelectricity with properly adjusted flexoelectric coefficients the pronounced low frequency decrease of the conductive s-EC threshold could be reproduced in a quantitative agreement with the experimental data.

Unfortunately, a similar quantitative match could not be achieved for dielectric s-EC patterns. Nevertheless, simulation experiments have shown that even the abrupt increase of the dielectric $U_{\mathrm{c}}(f)$ in thin cells at low $f$ can be qualitatively reproduced by the extended SM if one uses (together with the same flexoelectric coefficients as for the conductive s-EC) a $\sigma_{a} / \sigma_{\perp}$ value which is lower than expected from the measurements. One has to emphasize here that the fitted $e_{1}$ and $e_{3}$ parameters fall into the range of values measured for nematics, however, we do not claim that the fitted values are unique, some other $e_{1}, e_{3}$ combinations might provide almost as good match with experimental data. Furthermore, in the numerical calculations only a subset of parameters $\left(\sigma_{a}, \sigma_{\perp}, e_{1}, e_{3}\right)$ have been varied. One can not exclude that by slight adjustment of the other parameters (allowed by experimental errors in measuring the values in Table I) a better agreement between measurements and calculations could be obtained.

The numerical simulations presented above have confirmed that the experimentally detected novel low frequency dependencies of $U_{\mathrm{c}}(f)$ are consequences of the flexoelectricity and represent a competition between flexoelectric and Coulomb charge separation mechanisms. The frequency range in which it occurs is in the order of $1 / \tau_{\mathrm{d}}$, thus it can be tuned by the proper choice of the sample thickness $d$.

In the paper we have focused on the EC threshold voltages only. For a complete analysis of the onset behavior one should also confront the experimental and theoretical data regarding the critical wave-vector $\mathbf{q}_{c}$ of the pattern. The special experimental conditions, namely the small wavelength of the pattern (about the same - few microns - for conductive and dielectric s-EC as well as for nsEC), the fast traveling speed of the rolls, the relatively weak contrast (weaker than in thick samples) unfortunately did not allow to obtain $\mathbf{q}_{c}$ values with required precision by polarizing microscopy.

From the misfit of the dielectric thresholds one can draw a double conclusion. On the one hand, the obtained qualitative agreement may indicate that the extended SM does contain the main ingredients of the physical mechanism responsible for the formation of dielectric patterns even under our experimental conditions. On the other hand, however, the lack of quantitative match implies that the applicability limits of the model could be approached by testing thin cells at low frequencies and inclusion of additional phenomena (resulting in an apparent reduction of $\sigma_{a} / \sigma_{\perp}$ ) would be needed for a complete description. One such candidate could be the (unipolar) charge injection at the electrodes which is not included in the SM. For a low value of the overall conductivity (as in our case for dielectric s-EC) the weak charge focusing effect may be disrupted by charge injection at dc or at low frequency ac fields as it has been suggested recently 56.

We also have to note here that in our thin samples for all compounds traveling EC rolls have been detected at the onset. It has been proven that traveling waves can 
be explained if the assumption of Ohmic conductivity is given up and the SM is replaced by the weak electrolyte model (WEM) 55] taking into account ionic phenomena. It has also been shown that for typical EC samples $(d \gtrsim 10 \mu \mathrm{m})$ in the conductive s-EC regime the $U_{\mathrm{c}}$ and $\mathbf{q}_{c}$ values provided by the WEM differ by less than $1 \%$ from those calculated with the SM [34]. However, in this comparison flexoelectricity has not been included. Neither has the test been performed for smaller $d$ values, nor for dielectric s-EC. Therefore, a more complete description of the observed phenomena would need the inclusion of the flexoelectric effect into the WEM, and an extension of WEM based numerical studies to dielectric s-EC as well as to ns-EC, which is going far beyond the scope of the present paper. Such simulations would require some knowledge about additional material parameters, such as ionic mobility, recombination rate, equilibrium ion density. In this respect the measurements of the dc and low frequency ac conductivities of thin nematic layers could be important to clarify the impact of weak electrolyte effects. Future studies in these directions could possibly bridge the quantitative gap between the experiments and numerical data presented in this paper for the dielectric s-EC.

\section{Acknowledgments}

The authors thank to W. Pesch for fruitful discussions and for providing the numerical code. The authors are grateful to G. Pelzl for providing the liquid crystal 8/7. Financial support by the Hungarian Research Fund OTKA-K61075, DFG Grant No. Kr690/22-1 and SFB 481/A8 are gratefully acknowledged.
[1] L. Kramer and W. Pesch, in Pattern Formation in Liquid Crystals, edited by Á. Buka and L. Kramer (SpringerVerlag, New York, 1996), p. 221.

[2] L. Kramer and W. Pesch, in Physical Properties of Liquid Crystals: Nematics, edited by D.A. Dunmur, A. Fukuda, and G.R. Luckhurst (Inspec, London, 2001), p. 441.

[3] Á. Buka, N. Éber, W. Pesch, and L. Kramer, in Self Assembly, Pattern Formation and Growth Phenomena in Nano-Systems, edited by A.A. Golovin and A.A. Nepomnyaschy (Kluwer Academic Press, New York, 2006), p. 55.

[4] E.F. Carr, Mol. Cryst. Liq. Cryst. 7, 253 (1969).

[5] W. Helfrich, J. Chem. Phys. 51, 4092 (1969).

[6] S. Rasenat, G. Hartung, B.L. Winkler, and I. Rehberg, Exp. Fluids 7, 412 (1989).

[7] E. Bodenschatz, W. Zimmermann, and L. Kramer, J. Phys. France 49, 1875 (1988).

[8] W. Zimmermann, in Defects, Singularities and Patterns in Nematic Liquid Crystals, edited by J.M. Coron, J.M. Ghidaglia, and F. Helein, NATO Advanced Study Institute Series (Kluwer, Dordrecht, 1991), p. 401.

[9] B. Dressel, PhD Thesis, University of Bayreuth (2002).

[10] S. Rasenat, V. Steinberg, and I. Rehberg, Phys. Rev. A 42, 5998 (1990).

[11] U. Schneider, M. de la Torre Juarez, W. Zimmermann, and I. Rehberg, Phys. Rev. A 46, 1009 (1992).

[12] A.P. Krekhov, W. Pesch, N. Éber, T. Tóth-Katona, and Á. Buka, Phys. Rev. E 77, 021705 (2008).

[13] R.B. Meyer, Phys. Rev. Lett. 22, 918 (1969).

[14] P.G. de Gennes and J. Prost, The Physics of Liquid Crystals, (Clarendon Press, Oxford, 1993).

[15] S. Chandrasekhar, Liquid Crystals, (University Press, Cambridge, 1992).

[16] I.G. Chistyakov and L.K. Vistin, Sov. Phys. Crystallogr. 19, 119 (1974).

[17] Yu.P. Bobylev and S.A. Pikin, Sov. Phys. JETP 45, 195 (1977).

[18] M.I. Barnik, L.M. Blinov, A.N. Trufanov, and B.A. Umanski, Sov. Phys. JETP 46, 1016 (1977).
[19] L. Kramer, E. Bodenschatz, W. Pesch, W. Thom, and W. Zimmermann, Liq. Cryst. 5, 699 (1989).

[20] W.Thom, W.Zimmermann, L.Kramer, Liq.Cryst. 4, 309 (1989).

[21] N.V. Madhusudana and V.A. Raghunathan, Liq. Cryst. 5, 1789 (1989).

[22] Here, we kept the notations for different modes as introduced in Ref. [12], where the full description of the model is given. In Ref. [8] notations of IA, IIA, IB and IIB have been used for modes I, II, III and IV, respectively.

[23] M. Goscianski and L. Léger, J. Phys. (France) 36, 231 (1975).

[24] L.M. Blinov, M.I. Barnik, V.T. Lazareva, and A.N. Trufanov, J. Phys. (France) 40, 263 (1979).

[25] E. Kochowska, S. Németh, G. Pelzl, and Á. Buka, Phys. Rev. E 70, 011711 (2004).

[26] T. Tóth-Katona, A. Cauquil-Vergnes, N. Éber, and Á. Buka, Phys. Rev. E 75, 066210 (2007).

[27] P. Kumar, S.N. Patil, U.S. Hiremath, and K.S. Krishnamurthy, J. Phys. Chem B 111, 8792 (2007).

[28] D. Wiant, J.T. Gleeson, N. Éber, K. Fodor-Csorba, A. Jákli, and T. Tóth-Katona, Phys. Rev. E 72, 041712 (2005).

[29] M.-G. Tamba, W. Weissflog, A. Eremin, J. Heuer, and R. Stannarius, Eur. Phys. J. E 22, 85 (2007).

[30] R. Stannarius and J. Heuer, Eur. Phys. J. E 24, 27 (2007).

[31] J. Harden, B. Mbanga, N. Éber, K. Fodor-Csorba, S. Sprunt, J.T. Gleeson, and A. Jákli, Phys. Rev. Lett. 97, 157802 (2006).

[32] A.P. Krekhov and W. Pesch (unpublished).

[33] H. Kresse, A. Wiegeleben, and D. Demus, Krist. Tech. 15, 341 (1980).

[34] M. Treiber, N. Éber, Á. Buka, and L. Kramer, J. Phys. II France 7, 649 (1997).

[35] A. Hertrich, W. Decker, W. Pesch, and L. Kramer, J. Phys. II France 2, 1915 (1992).

[36] S.-Q. Zhou, N. Éber, Á. Buka, W. Pesch, and G. Ahlers, Phys. Rev. E 74, 046211 (2006). 
[37] Data sheet "N5 Licrystal" from Merck, Darmstadt, Germany (1982).

[38] H.H. Graf, H. Kneppe, and F. Schneider, Mol. Phys. 77, 521 (1992).

[39] W.H. de Jeu, W.A.P. Claasen, and A.M.J. Spruijt, Mol. Cryst. Liq. Cryst. 37, 269 (1976).

[40] F. Rondelez, D. Diguet, and G. Durand, Mol. Cryst. Liq. Cryst. 15, 183 (1971).

[41] G.J. Sprokel, Mol. Cryst. Liq. Cryst. 22, 249 (1973).

[42] E.J. Sinclair and E.F. Carr, Mol. Cryst. Liq. Cryst. 37, 303 (1976).

[43] H. Kneppe, F. Schneider, and N.K. Sharma, J. Chem. Phys. 77, 3203 (1982).

[44] C. Gähwiller, Phys. Lett. A 36, 311 (1971).

[45] L.M. Blinov L.A. Beresnev, S.A. Davydyan, S.G. Kononov, and S.V. Yablonski, Ferroelectrics 84, 365 (1988).

[46] I. Dozov, Ph. Martinot-Lagarde, and G. Durand, J. Phys. Lett. (France) 43, L-365 (1982).

[47] T. Takahashi, S. Hashidate, H. Nishijou, M. Usui, M. Kimura, and T. Akahane, Jpn. J. Appl. Phys. 37, 1982 (1998).

[48] A.G. Petrov, in Physical Properties of Liquid Crystals:
Nematics, edited by D.A. Dunmur, A. Fukuda, and G.R. Luckhurts (Inspec, London, 2001), p. 251.

[49] N. Éber, S.A. Rozanski, S. Németh, Á. Buka, W. Pesch, and L. Kramer, Phys. Rev. E 70, 061706 (2004).

[50] W. Pesch, L. Kramer, N. Éber, and Á. Buka, Phys. Rev. E 73, 061705 (2006).

[51] Y. Marinov, J. Kosmopoulos, W. Weissflog, A.G. Petrov and D.J. Photinos, Mol. Cryst. Liq. Cryst. 357, 221 (2001).

[52] O.A. Scaldin, A.N. Lachinov, and A.N. Chuvyrov, Sov. Phys.-Solid State 27, 734, (1985).

[53] O.A. Scaldin, and A.N. Chuvyrov, Sov. Phys.Crystallogr. 35, 505, (1990).

[54] Experimental results on a Phase 4 sample with $d \approx 11 \mu \mathrm{m}$ - see M. May, W. Schöpf, and I. Rehberg, Presentation at 35th Topical Meeting on Liquid Crystals, Bayreuth (2007), Book of Abstracts p. P38.

[55] M. Treiber and L. Kramer, Mol. Cryst. Liq. Cryst. 262, 311 (1995).

[56] K.S. Krishnamurthy and P. Kumar, Phys. Rev. E 76, 051705 (2007). 